\title{
Overview of 17,856 Compound Screening for Translation Inhibition and DNA Damage in Bacteria
}

\author{
P.V. Sergiev, E.S. Komarova (Andreianova), I.A. Osterman, \\ Ph. I. Pletnev, A. Ya. Golovina, I.G. Laptev, S.A. Evfratov, \\ E.I. Marusich, M.S. Veselov, S.V. Leonov, Ya. A. Ivanenkov, \\ A.A. Bogdanov and O.A. Dontsova
}

\begin{abstract}
Screening for new antibacterial compounds is an urgent need of medicinal chemistry. Understanding new antibiotics mechanism of action is needed for progression in the drug development pipeline. In the frame of the project supported by the Ministry of Science, we developed a reporter system which allows
\end{abstract}

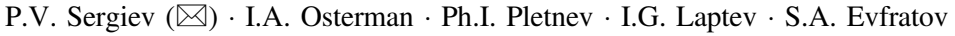
Department of Chemistry, Lomonosov Moscow State University, Moscow, Russia e-mail: petya@genebee.msu.ru
I.A. Osterman
e-mail: osterman@yandex.ru
Ph.I. Pletnev
e-mail: philippletnev@gmail.com

I.G. Laptev

e-mail: whiteswan92@gmail.com

S.A. Evfratov

e-mail: evfratov@gmail.com

E.S. Komarova (Andreianova)

Department of Bioengineering and Bioinformatics,

Lomonosov Moscow State University, Moscow, Russia

e-mail: ekaandreyanova@yandex.ru

A.Ya. Golovina

Belozersky Institute of Physico-Chemical Biology, Lomonosov Moscow

State University, Moscow, Russia

e-mail: malanka@yandex.ru

E.I. Marusich · M.S. Veselov - S.V. Leonov - Ya.A. Ivanenkov

Moscow Institute of Physics and Technology, Moscow, Russia

e-mail: mei@pharmcluster.ru

M.S. Veselov

e-mail: veselovmark@gmail.com 
an express, cost-effective and high-throughput screening for simultaneous detection of antibacterial activity, protein synthesis inhibition and induction of DNA damage SOS response. Automation of the screening process developed in the frame of this project allowed to screen up to 17,856 compound chemical library, supplied by the industrial partner of the project, Research Institute of Chemical Diversity. Among the tested compounds, DNA damaging agents appeared almost sixfold more frequently than those that inhibited protein synthesis. Several new families of antibacterial compounds were found among the tested set.

Keywords Antibiotic - Inhibitor - Ribosome $\cdot$ Protein synthesis Bacteria $\cdot$ Translation $\cdot$ Reporter strain

After initial success of antibiotics discovery [4, 21], the problem of bacterial infections appeared to be solved. In a golden period of antibiotics, lasted from 1940 s to 1960 s many diverse families of natural antibacterial compounds were discovered and applied in clinical practice [8]. However, the first antibiotic resistant bacteria were revealed shortly after [25]. Since that time, the frequency of new antibacterial compound discovery is decreasing dramatically [8]. At the same time, the antibiotic resistance isolates of pathogenic bacteria are spreading and even microbes resistant to multiple antibiotics and more recently totally resistant bacteria are found [1].

The standard pipeline of antimicrobial drug discovery starts with screening for antibacterial activity, most often in a culture broth of soil microorganisms. Following initial demonstration of the activity, an active compound needs to be purified to homogeneity and its mechanism of action should by studied ab initio. Often, and in the recent years even predominantly, application of this pipeline results in rediscovery of known antibacterials $[3,8,16]$. The time spent on the purification of the active compound and ab initio studies of the mechanism of action make the whole procedure lengthy, costly and inefficient.

The problems in the application of the standard pipeline noticed by both academic scientists and large pharmaceutical companies abandoned the area of new antimicrobials discovery [8]. However, the problem of antibiotic resistance remains

S.V. Leonov

e-mail: sl@pharmcluster.ru

Ya.A. Ivanenkov

e-mail: yai@pharmcluster.ru

A.A. Bogdanov · O.A. Dontsova

Department of Chemistry and Belozersky Institute of Physico-Chemical Biology,

Lomonosov Moscow State University, Moscow, Russia

e-mail: bogdanov@genebee.msu.ru

O.A. Dontsova

e-mail: olga.a.dontsova@gmail.com 
and growing worse year by year. Fresh ideas are needed to speed up antimicrobial compounds discovery. One of the possibilities is to combine initial screening for antibacterial activity with the built-in procedure to assess the mechanism of action [22]. High-throughput automation combined with the cost-efficient mechanism of action testing creates a possibility to revive antibacterial compounds discovery pipeline.

Usually, determination of the mechanism of action is time-consuming, expensive and not suitable for automation [22]. For example, the standard way is to test for the efficiency of incorporation of radioactive precursor molecules into DNA, RNA, and proteins. Experiments of this type require specialized protective environment to grow bacteria in radioactive culture medium and could not be done in a high-throughput form. Replacement of the radioactive precursor molecules with fluorescent ones [13] creates some advantage, but it is hardly suitable for initial screening due to the multistep lengthy implementation and the cost of reagents per test. A set of in vitro tests for partial reactions of protein biosynthesis is valuable for the screening of inhibitors [9], but very costly if applied to the high-throughput screening, due to the cost of the reagents and multistep procedure of the reaction setup.

An alternative to these methods is application of the reporter strains that respond to particular functional type of the inhibitor by upregulation of the gene, whose product is easy to detect $[14,22]$. Among the set of possible reporter genes, those coding for fluorescent proteins are preferable due to the lack of any reagent requirements, making application of other reporters, such as luciferases [18], much more expensive. Several reporter systems designed to detect particular classes of antibiotics are available. For example, there are reporters aimed in detection of beta lactams [24], tetracyclines [7], and macrolides [2, 11]. Detection of specific classes of known antibacterials is of use, but it is not applicable for the discovery of new antibacterials [14, 22].

Previously in our laboratory, a reporter plasmid was created that was based on the application of modified tryptophan attenuator as a sensor for translation inhibitors [12]. The attenuator of transcription preceding the genes coding for tryptophan biosynthesis pathway was discovered by C. Yanofsky group in 1970s [26]. Ribosome slows down on the doublet of tryptophan codons in a course of leader peptide synthesis results in the formation of antiterminator secondary structure in between the ribosome and RNA polymerase. In contrast, steady and quick translation in the presence of sufficient concentration of tryptophanyl-tRNA results in the formation of terminator hairpin in the nascent transcript, leading to premature termination of transcription preventing expression of tryptophan biosynthesis genes. We substituted tryptophan codons with those coding for abundant aminoacid alanine and introduced compensatory changes to the RNA in order to preserve a formation of alternative regulatory secondary structures [12]. These mutations made attenuator insensitive to the concentration of tryptophan. Ribosome interacting antibiotics may stall translation while the ribosome traverse leader region of attenuator. This stalling would cause folding of antiterminator RNA structure resulting in upregulation of the following gene. 
In the original reporter construct, we introduced CER fluorescent protein [20] downstream from the genetically modified tryptophan attenuator. Unregulated RFP fluorescent protein [10] was used as a control. This reporter construct was previously successfully applied for the screening of soil microorganisms culture broths provided by Gauze Institute of new antibiotics search [12]. This work resulted in identification of the mechanism of action for antibiotic amicoumacin A [17]. Antibacterial activity of this antibiotic was described previously [6], however, its mechanism of action was revealed only in our study [17].

In the frame of the current project supported by the Ministry of science, we improved the reporter construct. Due to the high background fluorescence of the rich culture media in the spectral area of CER protein fluorescence, we replaced CER protein gene with the gene of far red fluorescent protein Katushka2S [5] under a control of modified tryptophan attenuator (Fig. 1a). To test for two potential mechanisms of action simultaneously, we inserted Escherichia coli sulA promoter [23] in front of the RFP protein (Fig. 1a). This promoter is regulated by LexA transcriptional repressor, which is inactivated upon induction of the DNA damage SOS response [19]. The cells transformed by the resulting plasmid pDualrep2 [15] became a sensor for translation inhibitors which might be monitored by induction of Katushka2S expression and DNA damaging agents, such as topoisomerase II inhibitors, monitored by induction of RFP expression. These proteins possess readily distinguishable spectral properties that allow their separate detection via fluorescence scanner. A typical translation inhibitor erythromycin induces Katushka2S expression, while levofloxacin, an inhibitor of topoisomerase, induces expression of RFP (Fig. 1b). A number of antibiotics with known mechanism of action, such as erythromycin, roxithromycin, azithromycin, sulfanilamide, polymyxin, rifampicin, chloramphenicol, kanamycin, tetracycline, streptomycin,

(a)
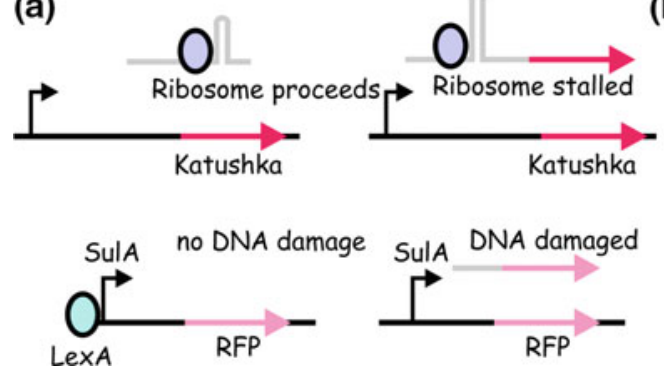

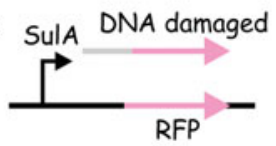

(b)

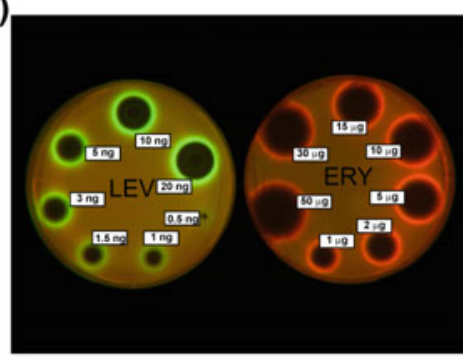

Fig. 1 Reporter system for detection of ribosome stalling and DNA damaging compounds. a Scheme of the reporter. Upper panel illustrates the organization of the sensor for translation inhibitors. On the left is the situation when ribosome is not inhibited. No Katushka2S expression is observed in this case. On the right is the situation when the ribosome is stalled by an antibiotic. In this case Katushka2S is upregulated. Lower panel corresponds to the sensor of DNA damage. On the left is situation when LexA represses transcription of the reporter in the absence of DNA damage. On the right is the situation when LexA is inactivated when DNA is damaged. In this case RFP is upregulated 
lincomycin, clindamycin, nalidixic acid, levofloxacin, ciprofloxacin, tobramycin, neomycin, etoposide, furagin, microcin B17, spectinomycin, etamycin A, hygromycin B, griseoviridin, tylosin, amicoumacin A, fusidic acid, puromycin, and gentamicin were tested against the created reporter strain [15]. It appeared that induction of RFP reporter is caused by all known topoisomerase inhibitors and in addition DNA precursor biosynthesis inhibitors. All substances that induced expression of Katushka2S belonged to translation inhibitors, although some ribosome-targeting antibiotics do not induce expression of the reporter gene. This result is expected, since some ribosome targeted antibiotics, such as aminoglycosides induce translation misreading, but not ribosome stalling.

The cells transformed by the pDualrep2 reporter were used for automated high throughput screening of the collection of 17,856 compounds provided by the industrial partner of the project, Research Institute of Chemical Diversity. The set was assembled from the core collection of Research Institute of Chemical Diversity aiming at maximization of chemical diversity. The screening was performed by automated liquid handling station Janus (Perkin Elmer). We spread the lawn of the E. coli reporter strain on top of $245 \mathrm{~mm} \times 245 \mathrm{~mm}$ square agar plate. The tested compounds were dissolved in DMSO to the concentration $17 \mathrm{mg} / \mathrm{ml}$. Total amount of $2 \mu \mathrm{l}$ volume of each obtained solution were spotted on agar plates by a 96-channel pipetting head of Janus liquid handling station (Perkin Elmer). After overnight growth at the $37{ }^{\circ} \mathrm{C}$, the plates are scanned by the fluorescence scanner (e.g., ChemiDoc, Bio-rad) at the wavelengths 553/574 nm (RFP) and 588/633 nm (Katushka2S). An example of a resulting plate scan is presented in Fig. 2a.

(a)

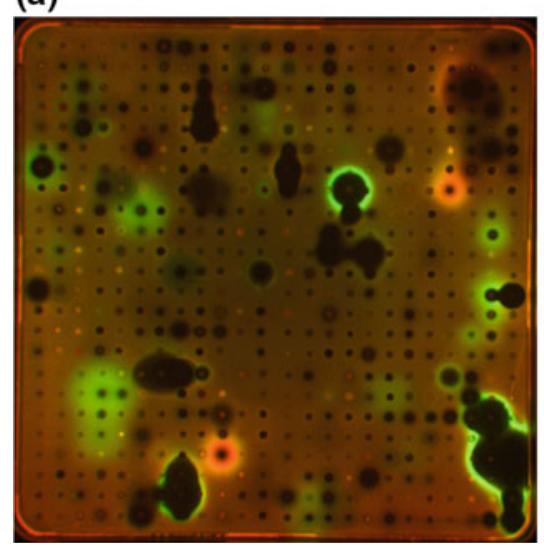

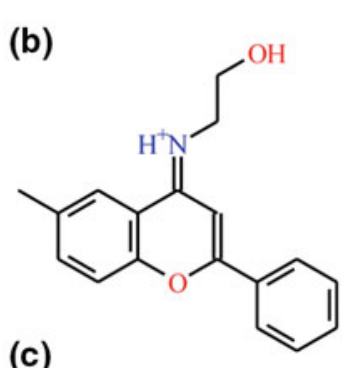

(c)<smiles>Cc1nc(NC(=N)N)nc2ccccc12</smiles>

Fig. 2 Results of the screening with the developed reporter system. a A sample of the screening plate with the 576 compounds applied onto the square agar plate with the reporter strain. The image is the superposition of the same plate scanned at 553/574 nm (RFP) presented as green pseudocolor and at 588/633 nm (Katushka2S) presented as red pseudocolor. b An example of the lead compound identified in the screening, 2-hydroxyethyl-(6-methyl-2-phenylchromen-4- ilyden) azanium. c Another lead compound identified in the screening, 4-methyl, 2-guanidino quinazoline 
In total, 184 compounds strongly inducing RFP and 32 compounds strongly inducing Katushka2S were found among the set of 17,856 compounds. Notably, DNA damaging agents are almost sixfold more abundant relative to translation inhibitors. Among the new lead compounds, we identified 2-hydroxyethyl(6-methyl-2-phenylchromen-4- ilyden) azanium (Fig. 2b) and 2-guanidino quinazoline derivatives (Fig. 2c). These lead structures will be further optimized aiming at the design of new more potent and selective antimicrobial substance.

\section{Conclusions}

The system for high throughput screening of antimicrobial compounds was created. The system allows classification of antibacterial compounds into stalling protein biosynthesis, inducing DNA damage and the compounds with the mechanism of action unrelated to these two. The system was successfully tested on a large set of the compounds. Several new classes of translation inhibitors were found.

Acknowledgement Researches are carried out (conducted) with the financial support of the state represented by the Ministry of Education and Science of the Russian Federation. Agreement (contract) no. 14.607.21.0086 06. Nov 2014. Unique project Identifier: RFMEFI60714X0086.

\section{References}

1. Almeida, Da Silva, P.E., Palomino, J.C.: Molecular basis and mechanisms of drug resistance in Mycobacterium tuberculosis: classical and new drugs. J. Antimicrob. Chemother. 66, 1417-1430 (2011)

2. Bailey, M., Chettiath, T., Mankin, A.S.: Induction of erm(C) expression by noninducing antibiotics. Antimicrob. Agents Chemother. 52, 866-874 (2008)

3. Fabbretti, A., Gualerzi, C.O., Brandi, L.: How to cope with the quest for new antibiotics. FEBS Lett. 585, 1673-1681 (2011)

4. Fleming, A.: On the antibacterial action of cultures of a penicillium, with special reference to their use in the isolation of B. influenzae. British J. Exp. Pathol. 10, 226-236 (1929)

5. Gurskaia, N.G., Staroverov, D.B., Fradkov, A.F., Luk'ianov, K.A.: Coding region of far-red fluorescent protein katushka contains a strong donor splice site. Bioorg. Khim. 37, 425-428 (2011)

6. Itoh, J., Omoto, S., Shomura, T., Nishizawa, N., Miyado, S., Yuda, Y., Shibata, U., Inouye, S.: Amicoumacin-A, a new antibiotic with strong antiinflammatory and antiulcer activity. J. Antibiot. 34, 611-613 (1981). (Tokyo)

7. Kurittu, J., Karp, M., Korpela, M.: Detection of tetracyclines with luminescent bacterial strains Lumin. J. Biol. Chem. Lumin. 15, 291-297 (2000)

8. Lewis, K.: Platforms for antibiotic discovery. Nat. Rev. Drug Discov. 12, 371-387 (2013)

9. Lowell, A.N., Santoro, N., Swaney, S.M., McQuade, T.J., Schultz, P.J., Larsen, M.J., Sherman, D.H.: Microscale adaptation of in vitro transcription/translation for high-throughput screening of natural product extract libraries. Chem. Biol. Drug Des. 86, 1331-1338 (2015)

10. Merzlyak, E.M., Goedhart, J., Shcherbo, D., Bulina, M.E., Shcheglov, A.S., Fradkov, A.F., Gaintzeva, A., Lukyanov, K.A., Lukyanov, S., Gadella, T.W.J., et al.: Bright monomeric red fluorescent protein with an extended fluorescence lifetime. Nat. Methods 4, 555-557 (2007) 
11. Möhrle, V., Stadler, M., Eberz, G.: Biosensor-guided screening for macrolides. Anal. Bioanal. Chem. 388, 1117-1125 (2007)

12. Osterman, I.A., Prokhorova, I.V., Sysoev, V.O., Boykova, Y.V., Efremenkova, O.V., Svetlov, M.S., Kolb, V.A., Bogdanov, A.A., Sergiev, P.V., Dontsova, O.A.: Attenuation-based dual-fluorescent-protein reporter for screening translation inhibitors. Antimicrob. Agents Chemother. 56, 1774-1783 (2012)

13. Osterman, I.A., Ustinov, A.V., Evdokimov, D.V., Korshun, V.A., Sergiev, P.V., Serebryakova, M.V., Demina, I.A., Galyamina, M.A., Govorun, V.M., Dontsova, O.A.: A nascent proteome study combining click chemistry with 2DE. Proteomics 13, 17-21 (2013)

14. Osterman, I.A., Bogdanov, A.A., Dontsova, O.A., Sergiev, P.V.: Techniques for Screening Translation Inhibitors. Antibiot. Basel Switz. 5, E22 (2016)

15. Osterman, I.A., Komarova, E.S., Shiryaev, D.I., Korniltsev, I.A., Khven, I.M., Lukyanov, D.A., Tashlitsky, V.N., Serebryakova, M.V., Efremenkova, O.V., Ivanenkov, Y.A., et al.: Sorting out antibiotics' mechanisms of action: a double fluorescent protein reporter for high throughput screening of ribosome and DNA biosynthesis inhibitors. Antimicrob. Agents Chemother. 60, 7481-7489 (2016)

16. Payne, D.J., Gwynn, M.N., Holmes, D.J., Pompliano, D.L.: Drugs for bad bugs: confronting the challenges of antibacterial discovery. Nat. Rev. Drug Discov. 6, 29-40 (2007)

17. Polikanov, Y.S., Osterman, I.A., Szal, T., Tashlitsky, V.N., Serebryakova, M.V., Kusochek, P., Bulkley, D., Malanicheva, I.A., Efimenko, T.A., Efremenkova, O.V., et al.: Amicoumacin a inhibits translation by stabilizing mRNA interaction with the ribosome. Mol. Cell. 56, 531-540 (2014)

18. Prokhorova, I.V., Osterman, I.A., Burakovsky, D.E., Serebryakova, M.V., Galyamina, M.A., Pobeguts, O.V., Altukhov, I., Kovalchuk, S., Alexeev, D.G., Govorun, V.M., et al.: Modified nucleotides $\mathrm{m}^{2} \mathrm{G} 966 / \mathrm{m}^{5} \mathrm{C} 967$ of Escherichia coli $16 \mathrm{~S}$ rRNA are required for attenuation of tryptophan operon. Sci. Rep. 3, 3236 (2013)

19. Reifferscheid, G., Buchinger, S.: Cell-based genotoxicity testing: genetically modified and genetically engineered bacteria in environmental genotoxicology. Adv. Biochem. Eng. Biotechnol. 118, 85-111 (2010)

20. Rizzo, M.A., Springer, G.H., Granada, B., Piston, D.W.: An improved cyan fluorescent protein variant useful for FRET. Nat. Biotechnol. 22, 445-449 (2004)

21. Schatz, A., Bugie, E., Waksman, S.A.: Streptomycin, a substance exhibiting antibiotic activity against gram-positive and gram-negative bacteria. Proc. Soc. Exp. Biol. Med. 55, 66-69 (1944)

22. Sergiev, P.V., Osterman, I.A., Golovina, A.Y., Laptev, I.G., Pletnev, P.I., Evfratov, S.A., Marusich, E.I., Leonov, S.V., Ivanenkov, Y.A., Bogdanov, A.A., et al.: Application of reporter strains for new antibiotic screening. Biomeditsinskaia Khimiia 62, 117-123 (2016)

23. Thomassen, G.O.S., Weel-Sneve, R., Rowe, A.D., Booth, J.A., Lindvall, J.M., Lagesen, K., Kristiansen, K.I., Bjørås, M., Rognes, T.: Tiling array analysis of UV treated Escherichia coli predicts novel differentially expressed small peptides. PloS one 5, E. 15356 (2010)

24. Valtonen, S.J., Kurittu, J.S., Karp, M.T.: A luminescent Escherichia coli biosensor for the high throughput detection of beta-lactams. J. Biomol. Screen. 7, 127-134 (2002)

25. Waksman, S.A., Reilly, H.C., Schatz, A.: Strain specificity and production of antibiotic substances: v. strain resistance of bacteria to antibiotic substances, especially to Streptomycin. Proc. Natl. Acad. Sci. USA 31, 157-164 (1945)

26. Zurawski, G., Elseviers, D., Stauffer, G.V., Yanofsky, C.: Translational control of transcription termination at the attenuator of the Escherichia coli tryptophan operon. Proc. Natl. Acad. Sci. USA 75, 5988-5992 (1978) 
Open Access This chapter is licensed under the terms of the Creative Commons Attribution 4.0 International License (http://creativecommons.org/licenses/by/4.0/), which permits use, sharing, adaptation, distribution and reproduction in any medium or format, as long as you give appropriate credit to the original author(s) and the source, provide a link to the Creative Commons license and indicate if changes were made.

The images or other third party material in this chapter are included in the chapter's Creative Commons license, unless indicated otherwise in a credit line to the material. If material is not included in the chapter's Creative Commons license and your intended use is not permitted by statutory regulation or exceeds the permitted use, you will need to obtain permission directly from the copyright holder.

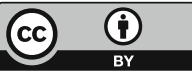

